\title{
NON-MATRIX POLYNOMIAL IDENTITY ENVELOPING ALGEBRAS
}

\author{
HAMID USEFI
}

\begin{abstract}
Let $L$ be a restricted Lie superalgebra with its restricted enveloping algebra $u(L)$ over a field $\mathbb{F}$ of characteristic $p>2$. A polynomial identity is called non-matrix if it is not satisfied by the algebra of $2 \times 2$ matrices over $\mathbb{F}$. We characterize $L$ when $u(L)$ satisfies a non-matrix polynomial identity.
\end{abstract}

\section{INTRODUCTION}

A variety of associative algebras over a field $\mathbb{F}$ is called non-matrix if it does not contain $M_{2}(\mathbb{F})$, the algebra of $2 \times 2$ matrices over $\mathbb{F}$. A polynomial identity (PI) is called non-matrix if $M_{2}(\mathbb{F})$ does not satisfy this identity. Latyshev in his attempt to solve the Specht problem proved that any non-matrix variety generated by a finitely generated algebra over a field of characteristic zero is finitely based [L77, L80]. The complete solution of the Specht problem in the case of characteristic zero is given by Kemer [K91].

Although several counterexamples are found for the Specht problem in the positive characteristic [AK], the development in this area has lead to some interesting results. Kemer in [K96] investigated the relation between PI-algebras and nil algebras and asked whether the Jacobson radical of a relatively free algebra of countable rank over an infinite field of positive characteristic is a nil ideal of bounded index. Amitsur had already proved in $\mathrm{Am}$ that the Jacobson radical of a relativelyfree algebra of countable rank is nil and Samoilov in Sam proved that the Jacobson radical of a relatively free algebra of countable rank over an infinite field of positive characteristic is a nilideal of bounded index. The non-matrix varieties have been further studied in BRT, MPR, R97.

Enveloping algebras satisfying polynomial identities were first considered by Latyshev [L63 by proving that the universal enveloping algebra of a Lie algebra $L$ over a field of characteristic zero satisfies a

Date: September 16, 2021.

2000 Mathematics Subject Classification. 16R10, 16R40, 17B35, 17B50.

Research was supported by NSERC. 
PI if and only if $L$ is abelian. Latyshev's result was extended to positive characteristic by Bahturin [B74]. Passman [P] and Petrogradsky [P91] considered the analogous problem for restricted Lie algebras and their envelopes.

Let $L=L_{0} \oplus L_{1}$ be a restricted Lie superalgebra with the bracket (, ). We denote the restricted enveloping algebra of $L$ by $u(L)$. All algebras in this paper are over a field $\mathbb{F}$ of characteristic $p>2$ unless otherwise stated. In case $p=3$ we add the axiom $((y, y), y)=0$, for every $y \in L_{1}$. This identity is necessary to embed $L$ in $u(L)$. Restricted Lie superalgebras whose enveloping algebras satisfy a polynomial identity have been characterized by Petrogradsky [P92]. The purpose of this paper is to characterize restricted Lie superalgebras whose restricted enveloping algebras satisfy a non-matrix PI. Riley and Wilson considered similar conditions for restricted enveloping algebras and group algebras in [RW]. Recall that a subset $X \subseteq L_{0}$ is called $p$-nilpotent if there exists an integer $s$ such that $x^{p^{s}}=0$, for every $x \in X$. Our main result is as follows.

Main Theorem. Let $L=L_{0} \oplus L_{1}$ be a restricted Lie superalgebra over a perfect field and denote by $M$ the subspace spanned by all $y \in L_{1}$ such that $(y, y)$ is p-nilpotent. The following statements are equivalent:

(1) $u(L)$ satisfies a non-matrix PI.

(2) $u(L)$ satisfies a PI, $\left(L_{0}, L_{0}\right)$ is p-nilpotent, $\operatorname{dim} L_{1} / M \leq 1$, $\left(M, L_{1}\right)$ is p-nilpotent, and $\left(L_{1}, L_{0}\right) \subseteq M$.

(3) The commutator ideal of $u(L)$ is nil of bounded index.

Theorem 2.6 below recalls Petrogradsky's characterization of when $u(L)$ satisfies an arbitrary PI strictly in terms of the underlying Lie superalgebra structure of $L$; this allows one to replace (2) with a similar such characterization (that is too cumbersome to state here). Furthermore, we show that (2) implies (3) over any field. However, given that $u(L)$ satisfies a non-matrix PI, the restriction on the field is necessary to be able to show that $\operatorname{dim} L_{1} / M \leq 1$. In Section 4 , we show that over a non-perfect field there exists a restricted Lie superalgebra $L=L_{0} \oplus L_{1}$ such that $\operatorname{dim} L_{1}=2$, commutator ideal of $u(L)$ is nil of index $2 p$ and yet $(y, y)$ is not $p$-nilpotent, for every $y \in L_{1}$. This is in complete contrast with the enveloping algebras of ordinary Lie superalgebras satisfying a non-matrix PI, see Theorem 1.2 of [BRU] where a similar characterization does not require any restriction on the field.

\section{Preliminaries}

Unless otherwise stated, all algebras are over a field $\mathbb{F}$ of characteristic $p>2$. Let $A=A_{0} \oplus A_{1}$ be a vector space decomposition of a 
non-associative algebra over $\mathbb{F}$. We say that this is a $\mathbb{Z}_{2}$-grading of $A$ if $A_{i} A_{j} \subseteq A_{i+j}$, for every $i, j \in \mathbb{Z}_{2}$ with the understanding that the addition $i+j$ is mod 2. The components $A_{0}$ and $A_{1}$ are called even and odd parts of $A$, respectively. Note that $A_{0}$ is a subalgebra of $A$. One can associate a Lie super-bracket to $A$ by defining $(x, y)=x y-(-1)^{i j} y x$ for every $x \in A_{i}$ and $y \in A_{j}$. If $A$ is associative, then for any $x \in A_{i}$, $y \in A_{j}$ and $z \in A$ the following identities hold:

(1) $(x, y)=-(-1)^{i j}(y, x)$,

(2) $(x,(y, z))=((x, y), z)+(-1)^{i j}(y,(x, z))$.

The above identities are the defining relations of Lie superalgebras. Furthermore, $A$ can be viewed as a Lie algebra by the usual Lie bracket $[u, v]=u v-v u$.

If $L$ is a Lie superalgebra, we denote the bracket of $L$ by $($,$) . The$ adjoint representation of $L$ is given by ad $x: L \rightarrow L$, ad $x(y)=(y, x)$, for all $x, y \in L$. The notion of restricted Lie superalgebras can be easily formulated as follows:

Definition 2.1. A Lie superalgebra $L=L_{0} \oplus L_{1}$ is called restricted, if there is a $p$ th power map $L_{0} \rightarrow L_{0}$, denoted as ${ }^{[p]}$, satisfying

(a) $(\alpha x)^{[p]}=\alpha^{p} x^{[p]}$, for all $x \in L_{0}$ and $\alpha \in \mathbb{F}$,

(b) $\left(y, x^{[p]}\right)=\left(y,,_{p} x\right)=(\operatorname{ad} x)^{p}(y)$, for all $x \in L_{0}$ and $y \in L$,

(c) $(x+y)^{[p]}=x^{[p]}+y^{[p]}+\sum_{i=1}^{p-1} s_{i}(x, y)$, for all $x, y \in L_{0}$ where $i s_{i}$ is the coefficient of $\lambda^{i-1}$ in $(\operatorname{ad}(\lambda x+y))^{p-1}(x)$.

For example, every $\mathbb{Z}_{2}$-graded associative algebra inherits a restricted Lie superalgebra structure.

Let $L$ be a restricted Lie superalgebra. We denote the (restricted) enveloping algebra of $L$ by $u(L)$. The augmentation ideal $\omega(L)$ is the ideal of $u(L)$ generated by $L$. The analogue of the PBW Theorem is as follows. We refer to [BMPZ] for basic background.

Theorem 2.2. Let $L=L_{0} \oplus L_{1}$ be a restricted Lie superalgebra and let $\mathcal{B}$ be a totally ordered basis for $L$ consisting of $\mathbb{Z}_{2}$-homogeneous elements. Then $u(L)$ has a basis consisting of $P B W$ monomials, that is, monomials of the form $x_{1}^{a_{1}} \ldots x_{s}^{a_{s}}$ where $x_{1}<\cdots<x_{s}$ in $\mathcal{B}, 0 \leq a_{i}<p$ whenever $x_{i} \in L_{0}$, and $0 \leq a_{i} \leq 1$ whenever $x_{i} \in L_{1}$.

Since $L$ embeds into $u(L)$ we identify $x^{[p]}$ with $x^{p}$, for every $x \in L_{0}$. Note that $u(L)$ can be viewed as a Lie algebra via the Lie bracket $[x, y]=x y-y x$ and if $x \in L_{0}$ and $y \in L$ then the bracket $(x, y)$ in $L$ is the same as the bracket $[x, y]$ in $u(L)$. Let $H$ be a subalgebra of $L$. We denote by $H^{\prime}$ the commutator subalgebra of $H$, that is $H^{\prime}=(H, H)$. For a subset $X \subseteq L$, we denote by $\langle X\rangle_{p}$ or $X_{p}$ the restricted ideal of $L$ 
generated by $X$. Also, $\langle X\rangle_{\mathbb{F}}$ denotes the subspace spanned by $X$. An element $x \in L_{0}$ is called $p$-nilpotent if there exists some non-negative integer $t$ such that $x^{p^{t}}=0$. Also, recall that $X$ is said to be $p$-nil if every element $x \in X$ is $p$-nilpotent and $X$ is $p$-nilpotent if there exists a positive integer $k$ such that $x^{p^{k}}=0$, for every $x \in X$. By an ideal of $L$ we always mean a restricted ideal, that is $I$ is an ideal of $L$ if $(I, L) \subseteq I$ and $I_{0}$ is closed under the $p$-map.

Let $B$ and $C$ be subspaces of $L$. We denote by $(B, C)$ the subspace spanned by all commutators $(b, c)$, where $b \in B$ and $c \in C$. The lower Lie central series of $L$ is defined by setting $\gamma_{1}(L)=L$ and $\gamma_{n}(L)=$ $\left(\gamma_{n-1}(L), L\right)$, for every $n \geq 2$. Recall that $L$ is called nilpotent if $\gamma_{n}(L)=0$, for some $n$. The derived subalgebra of $L$ is defined by setting $\delta_{0}(L)=L$ and $\delta_{i+1}(L)=\left(\delta_{i}(L), \delta_{i}(L)\right)$, for every $i \geq 0$. Also, $L$ is called solvable if $\delta_{m}(L)=0$, for some $m$, and the least of such $m$ is called the derived length of $L$. Moreover, long commutators are left tapped, that is $(x, y, x)=((x, y), z)$.

Note that Engel's Theorem holds for Lie superalgebras, see [Sch], for example.

Theorem 2.3 (Engel's Theorem). Let L be a finite-dimensional Lie superalgebra such that ad $x$ is nilpotent, for every homogeneous element $x \in L$. Then $L$ is nilpotent.

The proof of the following lemma follows from Engel's Theorem and the fact that $(\operatorname{ad} x)^{2}=\frac{1}{2} \operatorname{ad}(x, x)$, for every $x \in L_{1}$.

Lemma 2.4. Let $L=L_{0} \oplus L_{1}$ be a finite-dimensional restricted Lie superalgebra. If $L_{0}$ is $p$-nil then $L$ is nilpotent.

Lemma 2.5. Let $L$ be a restricted Lie superalgebra. Then $\omega(L)$ is associative nilpotent if and only if $L$ is finite-dimensional and $L_{0}$ is p-nil.

Proof. The if part follows from the PBW Theorem. We prove the converse by induction on $\operatorname{dim} L$. By Lemma 2.4, $L$ is nilpotent and so there exists a non-zero element $z$ in the center $Z(L)$ of $L$. Since $Z(L)$ is homogeneous we may assume that either $z \in L_{0}$ or $z \in L_{1}$. If $z \in L_{1}$ then $z^{2}=(z, z)=0$. If $z \in L_{0}$ then we can replace $z$ with its $p$-powers so that $z^{p}=0$. So in either case $z^{p}=0$ in $u(L)$. Now consider $H=L /\langle z\rangle_{p}$. Then by induction hypothesis $\omega(H)$ is nilpotent. This means that $\omega^{m}(L) \subseteq\langle z\rangle_{p} u(L)$, for some $m$. It then follows that $\omega^{m p}(L) \subseteq\left\langle z^{p}\right\rangle_{p} u(L)=0$, as required.

We shall use the following two results. 
Theorem 2.6 ([יP92]). Let $L=L_{0} \oplus L_{1}$ be a restricted Lie superalgebra. Then $u(L)$ satisfies a PI if and only if there exist homogeneous restricted ideals $B \subseteq A \subseteq L$ such that

(1) $L / A$ and $B$ are both finite-dimensional.

(2) $A^{\prime} \subseteq B, B^{\prime}=0$.

(3) The restricted Lie subalgebra $B_{0}$ is p-nilpotent.

Proposition 2.7 ([RW]). Let $R$ be an associative algebra that satisfies a non-matrix PI over a field of positive characteristic $p$. Then there exists an integer $t$ such that $R$ satisfies the identity $([u, v] w)^{p^{t}}=0$.

\section{PROOFS}

Proposition 3.1. Let $L$ be a restricted Lie superalgebra over a perfect field $\mathbb{F}$. Let $M$ be the set consisting of all $y \in L_{1}$ such that $(y, y)$ is p-nilpotent. If $u(L)$ satisfies a non-matrix PI then the following conditions are satisfied:

(1) $M$ is a subspace of $L_{1}$ and $\left(L_{1}, L_{0}\right) \subseteq M$;

(2) $\operatorname{dim} L_{1} / M \leq 1$;

(3) $\left(L_{0}, L_{0}\right)$ and $\left(M, L_{1}\right)$ are both p-nilpotent.

Proof. Let $R=[u(L), u(L)] u(L)$. Proposition 2.7 implies that $R$ is nil. Let $y \in M$ and $x \in L_{1}$. Note that $(x, y)=2 y x$ modulo $R$. So, $(y, x)^{2 m}=(y, y)^{m}(x, x)^{m}$ modulo $R$. Since $(y, y)$ is $p$-nilpotent and $R$ is nil, we deduce that $(x, y)$ is $p$-nilpotent. So, $\left(M, L_{1}\right)$ is $p$-nil. Let $y_{1}, y_{2} \in M$ and set $y=\alpha y_{1}+\beta y_{2}$, where $\alpha, \beta \in \mathbb{F}$. Note that $(y, y)^{p^{i}}=\left(y, \alpha y_{1}\right)^{p^{i}}+\left(y, \beta y_{2}\right)^{p^{i}}$ modulo $R$, for every $i$. Since $\left(M, L_{1}\right)$ and $R$ are both nil, we deduce that $(y, y)$ is $p$-nilpotent. This proves that $M$ is a subspace. Since $\left[L_{1}, L_{0}\right] \subseteq R$, we know by Proposition 2.7 that $\left[L_{1}, L_{0}\right]$ is nil. Thus, if $y \in\left[L_{1}, L_{0}\right]$ then $(y, y)=2 y^{2}$ is $p$-nilpotent. Hence, $\left[L_{1}, L_{0}\right] \subseteq M$ and this finishes the proof of (1).

Now, we show that $\operatorname{dim} L_{1} / M \leq 1$. Let $I$ be the subset of $L_{0}$ consisting of $p$-nilpotent elements. By Proposition 2.7, $I$ is a restricted ideal of $L_{0}$ and $\left(L_{0}, L_{0}\right) \subseteq I$. We have also proved that $\left(M, L_{1}\right) \subseteq I$. Note that $I+M$ is a restricted ideal of $L$. Without loss of generality, we can replace $L$ with $L /(I+M)$. So, $(y, y)$ is not $p$-nilpotent, for every $y \in L_{1}$. Let $y, z \in L_{1}$. Since $\left(L_{0}, L\right)=0$, we have

$$
[y, z]=-(y, z)+2 y z, \quad[y, z]^{2}=(y, z)^{2}-(y, y)(z, z) .
$$

By Proposition 2.7, there exists $m$ such that $[y, z]^{2 p^{m}}=0$. So,

$$
(y, z)^{2 p^{m}}=(y, y)^{p^{m}}(z, z)^{p^{m}} .
$$


Thus, by the PBW theorem, $(y, y)^{p^{m}}$ and $(z, z)^{p^{m}}$ must be linearly dependent. So, $(z, z)^{p^{m}}=\beta(y, y)^{p^{m}}$, for some $\beta \in \mathbb{F}$. Equation (1) then implies that

$$
\left((y, z)^{p^{m}}\right)^{2}=\beta\left((y, y)^{p^{m}}\right)^{2} .
$$

Using the PBW Theorem again, we deduce that $(y, y)^{p^{m}}=\alpha(y, z)^{p^{m}}$, for some $\alpha \in \mathbb{F}$. Equation (2) implies that $\beta \alpha^{2}=1$. So, we must have $(y, y)^{p^{m}}=\alpha(y, z)^{p^{m}}$ and $(z, z)^{p^{m}}=\alpha^{-1}(y, z)^{p^{m}}$. Let $\gamma \in \mathbb{F}$ so that $\gamma^{p^{m}}=\alpha$. We have, $(y, y-\gamma z)^{p^{m}}=0$ and $\left(z, z-\gamma^{-1} y\right)^{p^{m}}=0$. So, $(y-\gamma z, y-\gamma z)^{p^{m}}=0$ which implies that $y-\gamma z=0$. Thus, $y$ and $z$ are dependent, as required. This proves (2). In order to prove (3), it suffices to show that there exists an integer $m$ such that $\left(\left(L_{0}, L_{0}\right)+\left(M, L_{1}\right)\right)^{p^{m}}=0$. Note that, by Proposition 2.7, there exists an integer $t$ such that $[u, v]^{p^{t}}=0$, for all $u, v \in u(L)$. By Theorem [2.6, there exists a homogeneous ideal $A$ of $L$ of finite codimension such that $B=\left\langle A^{\prime}\right\rangle_{p}$ is finite dimensional and $B_{0}$ is $p$-nilpotent. We can replace $L$ with $L / B$. So we can assume that $A^{\prime}=0$. In particular, $A_{1} \subseteq M$. We claim that $L$ is solvable. Indeed, let $H=\left(L_{0}+M\right) / A$. It follows from Theorem 2.3 that $H^{\prime}$ is nilpotent. Thus, $H$ is solvable and, since $A$ is abelian, we deduce that $L_{0}+M$ is solvable. But $L /\left(L_{0}+M\right)$ is abelian which implies that $L$ is solvable, as claimed. Now, we argue by induction on the derived length $s$ of $L$. Suppose first that $L$ is metabelian, that is $\left(L^{\prime}, L^{\prime}\right)=0$. Since $\left(L_{0}, L_{0}\right)$ is abelian, it follows that $u^{p^{t}}=0$, for every $u \in\left(L_{0}, L_{0}\right)$. So, $\left(L_{0}, L_{0}\right)$ is $p$-nilpotent. Now we show that $\left(M, L_{1}\right)$ is $p$-nilpotent. Let $y_{1}, \ldots, y_{n}$ be linearly independent elements in $M$ so that their images span $M / A_{1}$. If $\operatorname{dim} L_{1} / M=1$, we take $z \in L_{1} \backslash M$. In part (1), we proved that $\left(M, L_{1}\right)$ is $p$-nil. Thus, there exists an integer $m>t$ such that $\left(y_{i}, y_{j}\right)^{p^{m}}=\left(z, y_{k}\right)^{p^{m}}=0$, for all $1 \leq i, j, k \leq n$. Then

$$
\begin{aligned}
\left(\sum_{i=1}^{n} \alpha_{i} y_{i}, \beta z+\sum_{j=1}^{n} \beta_{j} y_{j}\right)^{p^{m}} & =\left(\sum_{i=1}^{n} \sum_{j=1}^{n} \alpha_{i} \beta_{j}\left(y_{i}, y_{j}\right)+\sum_{i=1}^{n} \alpha_{i} \beta\left(y_{i}, z\right)\right)^{p^{m}} \\
& =\sum_{i=1}^{n} \sum_{j=1}^{n}\left(\alpha_{i} \beta_{j} y_{i}, y_{j}\right)^{p^{m}}+\sum_{i=1}^{n}\left(\alpha_{i} \beta y_{i}, z\right)^{p^{m}}=0
\end{aligned}
$$

where $\beta$ and the $\alpha_{i}$ and $\beta_{j}$ are in $\mathbb{F}$. On the other hand, let $y \in L_{1}$ and $x \in A_{1}$. Since $x^{2}=0$, we get

$$
[x, y]^{2}=(x, y)^{2}-2(x, y, y) x .
$$

Since $A^{\prime}=0$, we deduce that $[x, y]^{2 p}=(x, y)^{2 p}$. Thus, $(x, y)^{2 p^{t}}=$ $[x, y]^{2 p^{t}}=0$. We deduce that $\left(M, L_{1}\right)^{p^{m}}=0$. Since $L^{\prime}$ is abelian, 
it is clear that $\left(\left(L_{0}, L_{0}\right)+\left(M, L_{1}\right)\right)^{p^{m}}=0$. Now suppose $\delta_{s}(L)=0$, for some $s \geq 3$. Let $H=\left\langle\delta_{s-2}(L)\right\rangle_{p}$ and $K=\left\langle\delta_{s-1}(L)\right\rangle_{p}$. Note that $H_{1} \subseteq\left[L_{1}, L_{0}\right] \subseteq M$. Since $H$ is a metabelian ideal of $L$, we have $\left(\left(H_{0}, H_{0}\right)+\left(H_{1}, H_{1}\right)\right)^{p^{m}}=0$. On the other hand, by the induction hypothesis applied to $L / K$, we have $\left(\left(L_{0}, L_{0}\right)+\left(M, L_{1}\right)\right)^{p^{m}} \subseteq K_{0}$. But $K_{0}=\left\langle\left(H_{0}, H_{0}\right)+\left(H_{1}, H_{1}\right)\right\rangle_{p}$. So, we get $\left(\left(L_{0}, L_{0}\right)+\left(M, L_{1}\right)\right)^{p^{2 m}}=0$, as required.

Note that the difference between the restricted case and ordinary case mentioned in the introduction arises from Equation (1). In the ordinary Lie superalgebra case, discussed in [BRU], Equation (11) immediately implies that $(y, y)=\alpha(y, z)$ and $(z, z)=\alpha^{-1}(y, z)$, for some $\alpha \in \mathbb{F}$. We then deduce that $y$ and $z$ must be dependent and there is no need for the field to be perfect.

Lemma 3.2. Let $L$ be a restricted Lie superalgebra and $A$ a homogeneous ideal of $L$ of finite codimension. Let $I$ be an ideal of $u(A)$ that is stable under the adjoint action of $L$. If $u(L)$ is $P I$ and $I$ is nil of bounded index then so is $\operatorname{Iu}(L)$.

Proof. Let $R=u(L)$. Let $x_{1}, \ldots, x_{n} \in L_{0}$ and $y_{1}, \ldots, y_{m} \in L_{1}$ such that

$$
L=A+\left\langle x_{1}, \ldots, x_{n}, y_{1}, \ldots, y_{m}\right\rangle_{\mathbb{F}} .
$$

We assume that the $x_{i}$ and $y_{j}$ are linearly independent modulo $A$. By the PBW Theorem, $R$ has a basis consisting of the monomials of the form

$$
\begin{aligned}
& x_{1}^{\alpha_{1}} \ldots x_{n}^{\alpha_{n}} y_{1}^{\beta_{1}} \ldots y_{m}^{\beta_{m}} w \\
& 0 \leq \alpha_{i}<p, \quad \beta_{j} \in\{0,1\},
\end{aligned}
$$

where the $w$ 's are PBW monomials in $u(A)$. Let $D=u(A)$. Note that $R$ is a right $D$-module of finite rank $r=p^{n} 2^{m}$. Now consider the regular representation

$$
\rho: R \rightarrow \operatorname{End}\left(R_{D}\right)=M_{r}(D),
$$

where $\rho(u): R \rightarrow R$ is defined by $\rho(u) v=u v$, for every $u, v \in R$. Note that $\rho$ is injective because $R$ is unital. Thus, under $\rho$, we can embed $R$ into $M_{r}(D)$. Since $I$ is stable under the adjoint action of $L$, we have $R I=I R=R I R$. We claim that $R I$ embeds into $M_{r}(I)$. Indeed, let $v_{1} \in R I$ and $v_{2} \in R$. Since $\rho\left(v_{1}\right)\left(v_{2}\right)=v_{1} v_{2} \in R I$, we can write $v_{1} v_{2}$ as a linear combinations of elements of the form $u a$, where $u \in R$ and $a \in I$. So, each $u a$ and hence $v_{1} v_{2}$ is a linear combination of elements of the form $x_{1}^{\alpha_{1}} \ldots x_{n}^{\alpha_{n}} y_{1}^{\beta_{1}} \ldots y_{m}^{\beta_{m}} b$, where $b \in I$, as claimed. 
Therefore, it suffices to show that $M_{r}(I)$ is nil of bounded index. Recall Levitzki's Theorem and Shirshov's Height Theorem stating that every $t$-generated PI algebra which is nil of bounded index $s$ is (associative) nilpotent of a bound given as a function of $s, t$, and $d$, where $d$ is the degree of the polynomial identity, see [Lev] and [Sh]. So, if $S$ is any $r^{2}$-generated subalgebra of $I$ then there exists a constant $k$ such that $S^{k}=0$. Now, let $T \in M_{r}(I)$ and denote by $S$ the subalgebra of $I$ generated by all entries of $T$. So, $T^{i} \in M_{r}\left(S^{i}\right)$, for every $i$. Since $S^{k}=0$, we get $T^{k}=0$. Since $k$ is independent of $T$, it follows that $M_{r}(I)$ is nil of bounded index, as required.

Proof of the Main Theorem. The implication $(3) \Rightarrow(1)$ is obvious while $(1) \Rightarrow(2)$ follows from Proposition 3.1 . To prove $(2) \Rightarrow(3)$, we shall use the fact that the class of nil algebras of bounded index is closed under extensions. If $\operatorname{dim} L_{1} / M=1$ then we may assume that $L_{1}=M+\mathbb{F} z$, where $(z, z)$ is not $p$-nilpotent. By Theorem 2.6. there exists a homogeneous restricted ideal $A$ of finite codimension in $L$ such that $A^{\prime}$ is finite-dimensional and $\left(A^{\prime}\right)_{0}$ is $p$-nilpotent. Note that, by Lemma 2.4, $(A, A)$ is nilpotent. Thus, $\omega\left(\left\langle A^{\prime}\right\rangle_{p}\right)$ is associative nilpotent, by Lemma 2.5. Hence, $A^{\prime} u(L)$ is associative nilpotent. So, we can replace $L$ with $L /\left(A^{\prime}\right)_{p}$ to assume that $A$ is abelian. We claim that $\left(A_{1}, L_{1}\right)$ is $p$-nilpotent. If $M=L_{1}$, then $\left(L_{1}, L_{1}\right)$ is $p$-nilpotent and the claim is obvious. So we may assume that $(z, z)$ is not $p$-nilpotent and prove $A_{1} \subseteq M$. Suppose that there exists $x \in A_{1}$ such that $x \notin M$. Since $\operatorname{dim} L / M=1$, we may assume that $x=z+\alpha y$, for some $y \in M$ and $\alpha \in \mathbb{F}$. Then, since $(x, x)=0$, we get

$$
(z, z)=(x-\alpha y, x-\alpha y)=-2 \alpha(x, y)+\alpha^{2}(y, y)=\left(-2 \alpha x+\alpha^{2} y, y\right) .
$$

Since $y \in M$, it follows from the hypothesis that $\left(y, L_{1}\right)$ is $p$-nilpotent. So, $(z, z)$ must be $p$-nilpotent, which is a contradiction. Note that $u(A)$ is the tensor product of a commutative algebra with the Grassmann algebra. Thus, $u(A)$ satisfies $[x, y, z]=0$. So, we have $(x+y)^{p}=x^{p}+y^{p}$, for all $x, y \in u(A)$. Let $B=\langle(A, L)\rangle_{p}$. Note that $B$ is an abelian ideal of $L$ and since $\left(A_{0}, L_{0}\right)$ and $\left(A_{1}, L_{1}\right)$ are both $p$-nilpotent, $\omega(B)$ is nil of bounded index. It follows that $I=B u(A)$ is nil of bounded index. Furthermore, by Lemma $[3.2, I u(L)=B u(L)$ is also a nil ideal of $u(L)$ of bounded index. But $I u(L)$ is the kernel of the homomorphism $R \rightarrow u(L / B)$. Thus, we can replace $L$ with $L / B$ to assume that $A$ is central in $L$. It follows that $L^{\prime}$ is finite-dimensional. Note that $A_{1} u(A)$ is nil of index $p$. Since $A_{1}$ is a central ideal of $L, A_{1} u(L)$ is nil of bounded index, by Lemma 3.2. Thus, we may assume that $A_{1}=0$. It follows that $M$ is finite-dimensional. Thus, by Lemma 
2.5. $\left(\left(M, L_{1}\right)+M\right) u(L)$ is associative nilpotent. We can now assume $M=0$. Hence, $L_{1}=\mathbb{F} z$ and $\left(z, L_{0}\right)=0$. Let $H=L_{0}$. Since $H^{\prime}$ is finite-dimensional and $p$-nilpotent, we deduce, by Lemma 2.5 , that $\omega\left(\left\langle H^{\prime}\right\rangle_{p}\right)$ is associative nilpotent. So we can replace $L$ with $L /\left\langle H^{\prime}\right\rangle_{p}$. Hence, $\left(L_{0}, L\right)=0$. It then follows that $[R, R]=0$, as required.

\section{EXAMPLES}

We provide examples showing that the restriction on the field in the main result is necessary.

Example 4.1. Let $L=L_{0} \oplus L_{1}$ be a restricted Lie superalgebra over a non-perfect field $\mathbb{F}$, where $L_{0}=\left\langle x_{1}, x_{2}, x_{3}\right\rangle_{\mathbb{F}}$ and $L_{1}=\langle y, z\rangle_{\mathbb{F}}$. We assume that $\left(L_{0}, L\right)=0$ and set $x_{1}=(y, y), x_{2}=(z, z)$, and $x_{3}=$ $(y, z)$. Let $\alpha \in \mathbb{F}$ be an element whose pth root does not lie in $\mathbb{F}$. We define the p-mapping by setting $x_{1}^{p}=x_{1}, x_{2}^{p}=\alpha^{2} x_{1}$, and $x_{3}^{p}=\alpha x_{1}$. The following statements hold:

(1) The commutator ideal of $u(L)$ is nil of bounded index; hence $u(L)$ satisfies a non-matrix PI.

(2) $(c, c)$ is not p-nilpotent, for every $c \in L_{1}$.

Proof. Note that $[y, z]^{2}=x_{3}^{2}-x_{1} x_{2}$ is a central element in $u(L)$ and $[y, z]^{2 p}=0$. Every element in the commutator ideal of $u(L)$ is of the form $u=(\alpha y+\beta z+\gamma)[y, z]$, where $\alpha, \beta, \gamma$ are in the center of $u(L)$. We observe that

$$
\begin{aligned}
u^{2} & =(\alpha y+\beta z+\gamma)[y, z](\alpha y+\beta z+\gamma)[y, z] \\
& =(\alpha y+\beta z+\gamma)^{2}[y, z]^{2}+(\alpha y+\beta z+\gamma)[y, z, \alpha y+\beta z+\gamma][y, z] \\
& =(\alpha y+\beta z+\gamma)^{2}[y, z]^{2}+(\alpha y+\beta z+\gamma)(-2 \alpha y-2 \beta z)[y, z]^{2} \\
& =(\alpha y+\beta z+\gamma)(-\alpha y-\beta z+\gamma)[y, z]^{2}
\end{aligned}
$$

Thus $u^{2 p}=0$, for every $u \in[u(L), u(L)] u(L)$. Hence, the commutator ideal of $u(L)$ is nil of index $2 p$.

Next we prove (2). Suppose to the contrary that there exists $c \in$ $L_{1}$ such that $(c, c)$ is $p$-nilpotent. Without loss of generality, we may assume $c=y+\beta z$. We have

$$
[c, z]=-(c, z)+2 c z, \quad[c, z]^{2}=(c, z)^{2}-(c, c)(z, z) .
$$

Since $[c, z]^{2 p}=0$, we get $(c, z)^{2 p}=(c, c)^{p}(z, z)^{p}$. Since $(c, c)$ is $p$ nilpotent, $(c, z)$ must be $p$-nilpotent. So there exists an integer $m$ such 
that $(y+\beta z, z)^{p^{m}}=0$. Note that

$$
\begin{aligned}
(y+\beta z, z)^{p^{m}}=x_{3}^{p^{m}}+\beta^{p^{m}} x_{2}^{p^{m}} & =\left(\alpha x_{1}\right)^{p^{m-1}}+\beta^{p^{m}}\left(\alpha^{2} x_{1}\right)^{p^{m-1}} \\
& =\alpha^{p^{m-1}} x_{1}+\beta^{p^{m}} \alpha^{2 p^{m-1}} x_{1}=0
\end{aligned}
$$

We get $\beta^{p^{m}} \alpha^{p^{m-1}}+1=0$. Hence, $\left(\beta^{p} \alpha+1\right)^{p^{m-1}}=0$ which implies that $\alpha=(-\beta)^{p}$. So $\alpha$ must have $p$ th root in $\mathbb{F}$ which is a contradiction.

One might ask if similar examples exist when $\operatorname{dim} L_{1} \geq 3$. The answer is yes as the following example shows.

Example 4.2. Let $\mathbb{F} \subseteq K$ be a field extension and suppose that there exist $u, v \in K$ so that $\{1, u, v\}$ is linearly independent over $\mathbb{F}$ and $u^{p}, v^{p} \in \mathbb{F}$. For example, one can take $K=\mathbb{F}_{p}(u, v)$ and $\mathbb{F}=\mathbb{F}_{p}\left(u^{p}, v^{p}\right)$, where $\mathbb{F}_{p}$ is the prime field. Indeed, here we have $[K: \mathbb{F}]=p^{2}$.

We define a restricted Lie superalgebra $L=L_{0} \oplus L_{1}$ over $\mathbb{F}$ as follows. Let $L_{1}=\left\langle y_{1}, y_{2}, y_{3}\right\rangle_{\mathbb{F}}, L_{0}=\left\langle z_{i j}, x_{k} \mid 1 \leq i<j \leq 3,1 \leq k \leq 3\right\rangle_{\mathbb{F}}$ and assume that $\left(L_{0}, L\right)=0$. Set $z_{i j}=\left(y_{i}, y_{j}\right), x_{k}=\left(y_{k}, y_{k}\right)$, for all $1 \leq i<j \leq 3$ and $1 \leq k \leq 3$. Let $\alpha=u^{p}, \beta=v^{p}$ and define the p-mapping by $x_{1}^{p}=x_{1}, x_{2}^{p}=\alpha^{2} x_{1}, x_{3}^{p}=\beta^{2} x_{1}, z_{12}^{p}=\alpha x_{1}, z_{13}^{p}=\beta x_{1}$, and $z_{23}^{p}=\alpha \beta x_{1}$. The following statements hold:

(1) The commutator ideal of $u(L)$ is nil of bounded index.

(2) $(c, c)$ is not p-nilpotent, for every $c \in L_{1}$.

Proof. Let $J$ be the Jacobson radical of $u(L)$. So, $J$ is associative nilpotent. Let $A=L_{0} \oplus\left\langle y_{1}, y_{2}\right\rangle$ and set $P=u(A)$. Then, by Example 4.1. $[P, P] P$ is a nil ideal of $P$ of bounded index. So, by Lemma 3.2, we deduce that $[P, P] u(L)$ is nil of bounded index. Hence, $[P, P] u(L) \subseteq J$. We can similarly prove that $\left[y_{1}, y_{3}\right],\left[y_{2}, y_{3}\right] \in J$. This implies that the commutator ideal of $u(L)$ is nil of bounded index. This proves (1).

To prove (2), suppose to the contrary that there exists $c \in L_{1}$ such that $(c, c)$ is $p$-nilpotent. Without loss of generality, we may assume $c=y_{3}+\alpha_{1} y_{1}+\alpha_{2} y_{2}$. Then since the commutator ideal of $u(L)$ is nil of bounded index, $\left(y_{3}+\alpha_{1} y_{1}+\alpha_{2} y_{2}, y_{3}\right)$ is $p$-nilpotent. So there exists $m$ such that $\left(y_{3}+\alpha_{1} y_{1}+\alpha_{2} y_{2}, y_{3}\right)^{p^{m}}=0$. Note that

$$
\begin{aligned}
\left(y_{3}+\alpha_{1} y_{1}+\alpha_{2} y_{2}, y_{3}\right)^{p^{m}} & =x_{3}^{p^{m}}+\alpha_{1}^{p^{m}} z_{13}^{p^{m}}+\alpha_{2}^{p^{m}} z_{23}^{p^{m}} \\
& =\left(\beta^{2} x_{1}\right)^{p^{m-1}}+\alpha_{1}^{p^{m}}\left(\beta x_{1}\right)^{p^{m-1}}+\alpha_{2}^{p^{m}}\left(\alpha \beta x_{1}\right)^{p^{m-1}} \\
& =\beta^{p^{m-1}} x\left(\beta^{p^{m-1}}+\alpha_{1}^{p^{m}}+\alpha_{2}^{p^{m}} \alpha^{p^{m-1}}\right)=0 .
\end{aligned}
$$

We get $\left(\beta+\alpha_{1}^{p}+\alpha_{2}^{p} \alpha\right)^{p^{m-1}}=0$. Thus, $\beta+\alpha_{1}^{p}+\alpha_{2}^{p} \alpha=0$ and so $v+\alpha_{1}+\alpha_{2} u=0$. But this means that $u, v, 1$ are linearly dependent over $\mathbb{F}$, a contradiction. 


\section{REFERENCES}

[AK] E.V. Aladova, A.N. Krasilnikov, Polynomial identities in nil-algebras, Trans. Amer. Math. Soc. 361 (2009), no. 11, 5629-5646.

[Am] S.A. Amitsur, A generalization of Hilbert's Nullstellensatz, Proc. Amer. Math. Soc. 8 (1957), 649-656.

[BMPZ] Y. Bahturin, A. Mikhalev, V.M. Petrogradsky, M. Zaicev, Infinite Dimensional Lie Superalgebras, (Walter de Gruyter, Berlin, 1992).

[B74] Y. Bahturin, Identities in the universal envelopes of Lie algebras, J. Austral. Math. Soc. 18 (1974), 10-21.

[BRU] J. Bergen, D.M. Riley, H. Usefi, Lie superalgebras whose enveloping algebras satisfying a non-matrix polynomial identity, Israel J. Math, to appear.

[BRT] Y. Billig, D. Riley, V. Tasic, Nonmatrix varieties and nil-generated algebras whose units satisfy a group identity J. Algebra 190 (1997), no. 1, 241-252.

[K96] A.R. Kemer, PI-algebras and nil algebras of bounded index, Trends in Ring Theory, CMS Conf. Proc., Miskolc, (1996), (Amer. Math. Soc., Providence, 1998), 22, 59-69.

[K91] A.R. Kemer, Ideal of Identities of Associative Algebras, (Amer. Math. Soc., Providence, RI, 1991), Vol. 87.

[L80] V.N. Latyshev, Nonmatrix varieties of associative algebras, Mat. Zametki 27 (1980), no. $1,147-156$.

[L77] V.N. Latyshev, The complexity of nonmatrix varieties of associative algebras, I, II. Algebra i Logika 16 (1977), no. 2, 149-183, 184-199, 249-250.

[L63] V.N. Latyshev, Two remarks on PI-algebras, Sibirsk. Mat. Zh. 4 (1963), $1120-1121$.

[Lev] J. Levitzki, On a problem of A. Kurosch, Bull. Amer. Math. Soc. 52 (1946), 1033-1035.

[MPR] S.P. Mishchenko, V.M. Petrogradsky, A. Regev, Characterization of nonmatrix varieties of associative algebras, Israel $J$. Math. to appear.

[P] D.S. Passman, Enveloping algebras satisfying a polynomial identity, J. Algebra. 134(2) (1990), 469-490.

[P92] V.M. Petrogradski, Identities in the enveloping algebras for modular Lie superalgebras, J. Algebra 145 (1992), no. 1, 1-21.

[P91] V.M. Petrogradsky, The existence of an identity in a restricted envelope, Mat. Zametki 49(1) (1991), 84-93.

[RW] D.M. Riley, M.C. Wilson, Group algebras and enveloping algebras with nonmatrix and semigroup identities, Comm. Algebra 27 (7) (1999), 3545-3556.

[R97] D.M. Riley, PI-algebras generated by nilpotent elements of bounded index, J. Algebra 192 (1997), no. 1, 1-13.

[Sam] L.M. Samoilov, On the radical of a relatively free associative algebra over fields of positive characteristic, Mat. Sb. 199 (2008), no. 5, 81-126.

[Sch] M. Scheunert, The theory of Lie superalgebras, Lecture Notes in Math. 716 (1979).

[Sh] A.I. Shirshov, On rings with identity relations, Mat. Sb. 43 (85) (1957), 277283.

Department of Mathematics and Statistics, Memorial University of

Newfoundland, St. John's, NL, Canada, A1C 5S7

E-mail address: usefi@mun.ca 